\title{
INNOVACIÓN Y COMPETITIVIDAD EN MICROEMPRESAS PRODUCTORAS DE CERVEZA ARTESANAL EN MÉXICO ${ }^{30}$
}

\author{
$\underline{\text { Anastacio Espejel }}^{a^{*}}$, Ricardo Cruz $^{a}$ Ariadna Barrera ${ }^{a}$, Marco Ramírez ${ }^{a}$
}

${ }^{a}$ Universidad Autónoma Chapingo, Texcoco, Estado de México, México (aespejelg@chapingo.mx).

La cerveza es una bebida alcohólica de gran aceptación a nivel mundial y nacional; es elaborada con agua, cebada u otro grano malteado, lúpulo y levadura. En los últimos años en México, han surgido empresas productoras de cerveza artesanal impulsadas por consumidores que prefieren una cerveza con mejores atributos sensoriales. Estas empresas presentan diversas desventajas con respecto a su principal competencia, que es la gran industria. El objetivo de este trabajo fue analizar el nivel de adopción de innovaciones y la posición competitiva de una muestra exploratoria de cervecerías artesanales. Se aplicó una encuesta estructurada a diez empresas; la encuesta analizó la dinámica de la agroindustria, infraestructura, costos de producción, organización y dinámica de innovaciones. Los resultados sugieren que existen tres estratos de empresas con base al nivel de adopción de innovaciones (bajo con 24.3\% de adopción de innovaciones, medio con $35.0 \%$ y alto con $72.4 \%$ ). Las empresas con mejor posicionamiento competitivo poseen una alta correlación con adopción de innovaciones en calidad del producto, envasado y sustentabilidad, mientras que las del estrato medio adoptan innovaciones de proceso y mercado.

Palabras clave: Innovación, cervecería artesanal, consumidor, productos tradicionales, agroindustria

\section{Introducción}

La producción de cerveza equivale al $75 \%$ de la cuota del mercado global de bebidas alcohólicas, siendo los principales mercados: India, China, Estados Unidos, Brasil, Rusia, Alemania y México. Actualmente, de una lista de 125 países, México se encuentra en el séptimo lugar como productor, el primer lugar como exportador, y el $15^{\circ}$ lugar como importador de cerveza (Deloitte SL , 2017). Hoy en día, la cerveza mexicana tiene presencia en más de 180 países, con $21.3 \%$ de participación y aproximadamente 2,800 millones de dólares en ventas (World’s Richiest Countries, 2016). En América Latina, México es el tercer país en consumo de esta bebida, siendo el consumo de 60 litros de cerveza per cápita por año (Secretaría de Economía, 2015).

En años recientes se han modificado los gustos y preferencias de los consumidores de cerveza, la tendencia en el consumo de bebidas alcohólicas como la cerveza artesanal va a la alza a pesar de no contar con una definición precisa y oficial en México es considerada como aquella que prioriza el uso de materias primas de buena calidad; la mayor parte del proceso de elaboración se realiza de manera artesanal y por lo general se elabora en micro cervecerías, estas características confieren a este tipo de cerveza atributos sensoriales únicos, los cuales están por encima de la estandarizada cerveza industrial-comercial (Albán-Cabaco, et. al. 2015).

\section{Objetivo}

Analizar el nivel de adopción de innovaciones y la posición competitiva de una muestra exploratoria de cervecerías artesanales de México.

\section{Metodología}

Se aplicó una encuesta estructurada a una muestra exploratoria diez cervecerías de diferente nivel de desarrollo con el propósito de caracterizar la agroindustria en distintas regiones del país. La encuesta contenía los siguientes apartados 1) Dinámica de la agroindustria, con información relacionada a producción, proveeduría y comercialización; 2) Infraestructura, en donde se consideró las áreas y equipo con el que cuentan las empresas; 3) Costos de producción, se indagaron los costos e ingresos; 4) Organización, se investigó la afiliación a asociaciones o gremios de productores; y 5) Dinámica de innovaciones donde se evaluó la adopción de innovaciones relacionadas con análisis de calidad, sustentabilidad, prácticas de envasado, producción y mercado. Se creo un índice de adopción de adopción de innovaciones para cada categoría y se emplearon como estadísticos descriptivos para realizar una Clusterización Aglomerativa Jerárquica (CAJ) con disimilitud la distancia euclidiana, método de

${ }^{30}$ Proyecto 20352-C-62 financiando con fondos de la Dirección Genreral de Investigación y Posgrado de la Universidad Autónoma Chapingo. 
aglomeración Ward y truncando de clases a tres. Estos mismos valores se utilizaron para realizar un Análisis de Componentes Principales (ACP) y con ello analizar las correlaciones entre las observaciones (microcervecerias) y variables y con ello se definió la posición competitiva de cada empresa con base a la adopción de innovación.

\section{Resultados}

\subsection{Caracterización de la agroindustria de cerveza artesanal}

El número de cervecerías entrevistadas de manera exploratoria fueron diez.Los productores encuestados se caracterizaron por producir en promedio $572.1 \mathrm{~L}$ de cerveza artesanal por día, tener un rendimiento de $4 \mathrm{~L}$ por cada kilogramo de malta y contar con proveedores de Malta a empresas de EE. UU (6 empresas), Alemania (3), Holanda (1), Inglaterra (2), Chihuahua (1) y Puebla (1); de levadura empresas de EE. UU (6), Ciudad de México (1), Baja California Sur (1) y de lúpulo a empresas de EE. UU (6) Europa (1), Baja California (1), Baja California Sur (1), Chihuahua (1).

Se identificaron catorce problemas comunes, los cuales son considerados principales por los productores, tales como: dificultad para el abasto de insumos como malta y lúpulo (14.8\%), alta contaminación de las micro cervecerías (14.8\%) insuficiente capacidad del equipo (14.8\%), altos costos de producción (11.1\%), exceso de trámites burocráticos $(7.4 \%)$ y baja estandarización del proceso productivo (7.4\%). Como factores sociales, económicos, técnicos u organizacionales que los productores consideran de mayor impacto en la producción de cerveza se encuentra la mejora del equipo de producción (80 \%), disminución del costo de las materias primas (70 \%) la necesidad de capacitación y asistencia técnica (60\%), aumento de oferta de insumos ( $5 \%$ ), apoyos económicos y conocimiento del mercado (3\%) y mejor precio de venta $(1 \%)$.

Existe una gran diversidad entre los tipos de cerveza que ofertan este grupo de empresas, los estilos con mayor frecuencia en su elaboración corresponden a las cervezas estilo Pale Ale (7 empresas) cervezas oscuras (7), seguidas de IPA (6), trigo (5), claras (3), cervezas ámbar (2) y otros (4).

Con relación a la infraestructura, se encontró que un $30 \%$ de empresas tienen áreas definidas y diferenciadas, $10 \%$ con todas las áreas necesarias consolidadas mientras que el $60 \%$ está limitado en espacio debido a que la producción es realizada en los hogares de los productores. Las áreas con las que regularmente cuentan estas empresas son: recepción de materia prima (30\%), maduración (40\%), oficinas (40\%), almacén general (60\%), envasado (60\%), fermentación (70\%) y almacenamiento de producto final (70\%). Adicionalmente estas empresas cuentan con equipo básico que son molinos, maceradores, fermentadores y equipo de carbonatación, el $50 \%$ cuentan con cámara de refrigeración y $80 \%$ con barriles de acero inoxidable.

Los canales de comercialización mediante los cuales se distribuye la cerveza artesanal son directamente a consumidores y restaurantes; el precio promedio es de $\$ 72.9$ y $\$ 56.25$ pesos mexicanos, respectivamente. El $60 \%$ vende a clientes fijos como bares o restaurantes y el $40 \%$ lo hace directamente a turistas o consumidores interesados en degustar una cerveza artesanal. El ingreso principal del $70 \%$ de los propietarios proviene de la producción y venta de cerveza, mientras que el $30 \%$ restante proviene de otras fuentes de ingreso.

Uno de los problemas más relevantes a los que se enfrentan los microcerveceros es la monopolización de la materia prima por parte de la gran industria cervecera y con ello los elevados costos de producción asociados a los elevados precios de la materia prima y altos impuestos. Por otra parte, el $60 \%$ de las cervecerías están afiliadas a una asociación de la cual obtienen beneficios como la gestión de apoyos (66.6 $\%$ ), obtención de mejores precios (50 \%), reducción de los costos de producción (50\%), acceso a mercados más rentables $(50 \%)$ y asesoría y capacitación especializada $(50 \%)$.

\subsection{Adopción de innovaciones}

La innovación es una de las formas más estratégicas mediante la cual las microcervecerias permanecen en el mercado y en el gusto de los consumidores. Es un factor determinante para sobrevivir en un sector monopolizado por las cervecerías industriales y por cambios constantes en la preferencia de los consumidores. Con base a ello, se realizó una estratificación de las cervecerías considerandola adopción de 
tecnología; se realizó un análisis de Clusterización Aglomerativa Jerárquica del cual resultaron tres grupos de microcervecerias (Grafico 1).

Grafico 1. Dendrograma de cervecerías artesanales.

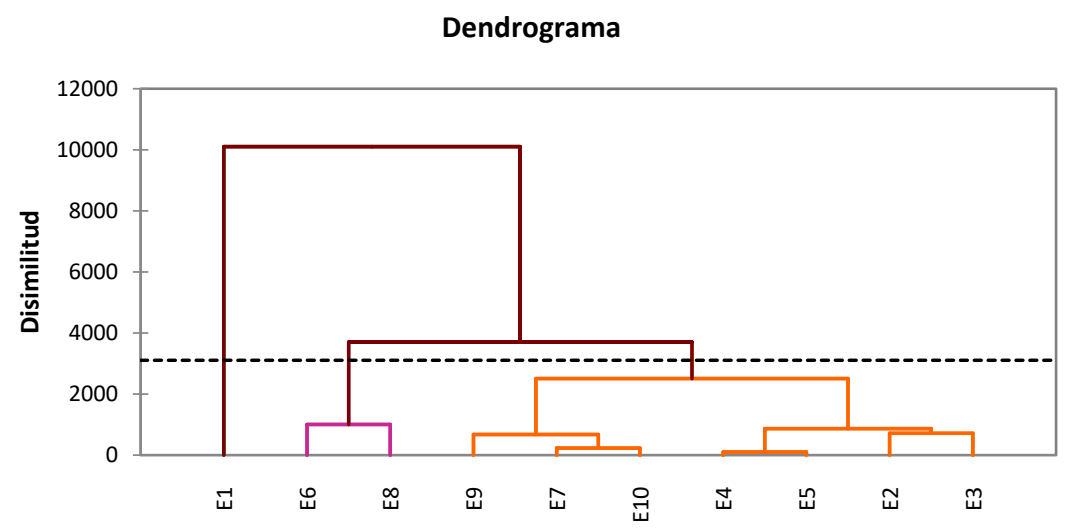

En el grupo 1 se ubica la empresa E1, con el mayor volumen de producción de cerveza y el mayor índice de adopción de innovaciones (Cuadro 1); en el grupo 2 se ubicaron las empresas E2, E3, E4, E5, E7 y E9, estas son las empresas de menor escala de producción y con el menor índice de adopción de innovaciones, con excepción de las categorías de mercado (58.3\%) y sustentabilidad (14.3\%) en comparación con el grupo 3 (54.2\% y $0 \%$ respectivamente).

Cuadro 1. Índice de adopción de innovación (promedio) por estrato.

\begin{tabular}{|c|c|c|c|c|c|c|c|}
\hline Estrato & Empresas integradas & Proceso & Análisis de calidad & Envasado & Mercado & Sustentabilidad & Promedio \\
\hline 1 & E1 & 78.6 & 66.7 & 75.0 & 91.7 & 50.0 & 72.4 \\
\hline 2 & $\begin{array}{l}\text { E2, E3, E4, E5, E7, E9 } \\
\text { у E10 }\end{array}$ & 42.9 & 2.4 & 3.6 & 58.3 & 14.3 & 24.3 \\
\hline 3 & E6 y E8 & 50.0 & 33.3 & 37.5 & 54.2 & 0.0 & 35.0 \\
\hline
\end{tabular}

Con respecto al análisis de componentes principales con un valor de KMO: 0.70 el cual es adecuado para este tipo de estudios; con dos componentes se explica el $79.26 \%$ (Cuadro 3) de la variabilidad de los datos.

Cuadro 3. Valores propios del Análisis de Componentes Principales

\begin{tabular}{lccccc}
\hline Factor: & F1 & F2 & F3 & F4 & F5 \\
\hline Valor propio & $\mathbf{2 . 9 5 2}$ & $\mathbf{1 . 0 1 0}$ & 0.614 & 0.247 & 0.176 \\
Variabilidad (\%) & 59.049 & 20.209 & 12.279 & 4.939 & 3.524 \\
\% acumulado & $\mathbf{5 9 . 0 4 9}$ & $\mathbf{7 9 . 2 5 8}$ & $\mathbf{9 1 . 5 3 7}$ & $\mathbf{9 6 . 4 7 6}$ & $\mathbf{1 0 0 . 0}$
\end{tabular}

Con respecto al grafico 2, se observan los vectores correspondientes al índice de innovación y las observaciones (E1... E10). Se destaca que los vectores de sustentabilidad son consistentes, pero no se correlacionan con las empresas, así como los vectores de mercado y proceso, esto sugiere que si bien son variables importantes, muestran una baja correlación con las empresas. La E1 con el mayor volumen e índice de innovación muestra alta correlación con calidad, envasado y en menor medida sustentabilidad; por su parte las empresas E2 y E6, pese a que están en el mismo cuadrante de los vectores de mercado y proceso su correlación es baja. La empresa con mejor posición competitiva es la E1, seguida de E2 y E6 y con la más baja posición el resto. 
Grafico 2. Biplot de cervecerías artesanales entrevistadas y variables de innovaciones.

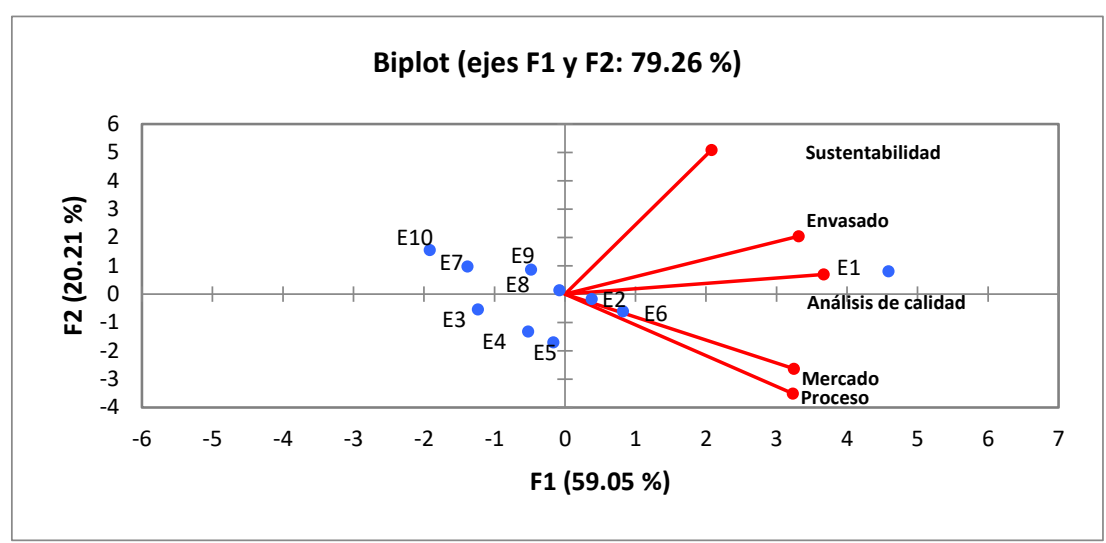

Estos resultados sugieren alta correlación entre el tamaño de las empresas con el índice de adopción de innovaciones, eso las ubica en una mejor posición. Con relación a las variables analizadas, existe una alta correlación entre mercado y proceso, lo cual sugiere que en la medida que una mejore la otra lo hará en el mismo sentido; de igual manera las variables de envasado y análisis de calidad (cuadro 4).

Cuadro 4. Matriz de correlaciones de Pearson

\begin{tabular}{llllll}
\hline Variables & Proceso & Análisis de calidad & Envasado & Mercado & Sustentabilidad \\
\hline Proceso & $\mathbf{1}$ & 0.586 & 0.468 & $\mathbf{0 . 7 3 6}$ & 0.106 \\
Análisis de calidad & 0.586 & $\mathbf{1}$ & $\mathbf{0 . 7 7 4}$ & 0.581 & 0.395 \\
Envasado & 0.468 & $\mathbf{0 . 7 7 4}$ & $\mathbf{1}$ & 0.383 & 0.420 \\
Mercado & $\mathbf{0 . 7 3 6}$ & 0.581 & 0.383 & $\mathbf{1}$ & 0.269 \\
Sustentabilidad & 0.106 & 0.395 & 0.420 & 0.269 & $\mathbf{1}$ \\
\hline
\end{tabular}

Los valores en negrita son diferentes de con un nivel de significancia de $p<0.05$

\section{Conclusiones}

Este fue un estudio exploratorio y estadísticamente no representativo de la agroindustria de cervecería artesanal. Las cervecerías analizadas se clasificaron en tres grupos de acuerdo con su nivel tecnológico. El primer grupo cuenta con la mayor adopción de innovaciones de proceso, envasado, calidad y sustentabilidad; en el segundo grupo se ubicaron las empresas de menor escala de producción y con menor adopción de innovaciones; el tercer grupo tiene un nivel intermedio de adopción, siendo las más importantes, las innovaciones de mercado y proceso.

La característica más común entre las empresas analizadas es el esfuerzo que realizan en cuanto a la adopción de innovaciones de mercado y proceso; la empresa con mejor posicionamiento competitivo se caracteriza por adoptar prácticamente todas las innovaciones, principalmente las de mercado y proceso, mientras que las de menor posicionamiento siguen una tendencia inversa a la empresa líder o mejor posicionada.

\section{Bibliografía}

Albán-Cabaco, B., Nuñez-Tabales, J. M., y Sánchez-Cañizares, S. M. (2015). "El sector cervecero artesanal español y sus posibilidades de internacionalización". Regional and Sectoral Economic Studies, 15(2): 146.

Deloitte SL. (2017). Cerveza artesanal: una experiencia multisensorial. Deloitte, México:

Secretaría de Economía. (2015). Industria de la cerveza en México. Secretaría de Economía.

World's Richiest Countries. (2016). Top Beer Exporters 2016. 